\title{
Quality of Care in Childhood-onset Systemic Lupus Erythematosus: Report of an Intervention to Improve Cardiovascular and Bone Health Screening
}

\author{
Emily A. Smitherman ${ }^{1}$ (D), Bin Huang ${ }^{2}$ (D) , Adam Furnier ${ }^{3}$, Janalee Taylor ${ }^{4}$, Mary Beth Burns ${ }^{4}$, \\ Hermine I. Brunner ${ }^{5}$ (D) and Esi M. Morgan ${ }^{6}$ (i)
}

\begin{abstract}
Objective. Initial benchmarking of childhood-onset systemic lupus erythematosus (cSLE) quality indicators revealed suboptimal performance across multiple centers. Our aim was to improve cardiovascular and bone health screenings at a tertiary treatment center for cSLE. This included annual measurements of vitamin D, lipid profiles, and bone mineral density through dual-energy $\mathrm{x}$-ray absorptiometry (DXA).

Methods. Quality improvement methodology was applied to design and implement a standardized previsit planning process to electronically entered and saved orders for needed screenings prior to a scheduled clinic visit. Process outcomes were measured using statistical process control charts. Univariate analyses were completed to assess patient-level factors.

Results. During the study, 123 patients with cSLE participated across 619 clinic visits. The percentage of patients with completed screenings improved from $54 \%$ to $92 \%$ for annual vitamin D, $55 \%$ to $84 \%$ for annual lipid profiles, and $57 \%$ to $78 \%$ for DXA, which was sustained for more than 1 year. Providers responded to a majority of abnormal results, and improvement in the average vitamin D level was observed over time. Higher levels of disease activity, damage, number of clinic visits, and screenings completed at baseline were observed in patients with all screenings completed at the end of the intervention.

Conclusion. Implementation of elements of the chronic illness care model for cSLE management improved performance of cardiovascular and bone health screenings, a step toward preventing longterm morbidity in cSLE. Our study also suggests that more patient interaction with the healthcare system may promote successful completion of health maintenance screenings.
\end{abstract}

Key Indexing Terms: bone density, cardiovascular diseases, disease prevention and control, pediatric systemic lupus erythematosus

Childhood-onset systemic lupus erythematosus (cSLE) is a chronic autoimmune disease with poor health outcomes and high rates of devastating disease complications, some of which may be preventable. Compared to patients with adult-onset disease, patients with cSLE, defined as disease onset before 18 years, have

${ }^{1}$ E.A. Smitherman, MD, MSCTR, Assistant Professor, Division of Pediatric Rheumatology, University of Alabama at Birmingham, Birmingham, Alabama; ${ }^{2}$ B. Huang, PhD, Professor, Division of Biostatistics and Epidemiology, Cincinnati Children's Hospital Medical Center, and Department of Pediatrics, University of Cincinnati College of Medicine, Cincinnati; ${ }^{3}$ A. Furnier, BS, Quality Improvement Consultant, James $M$. Anderson Center for Health Systems Excellence, Cincinnati Children's Hospital Medical Center, Cincinnati; ${ }^{4}$ J. Taylor, MSN, APRN, CNP, M.B. Burns, RN, CPN, Division of Rheumatology, Cincinnati Children's Hospital Medical Center, Cincinnati; ${ }^{5}$ H.I. Brunner, $M D, M S c, M B A$, Professor, Division of Rheumatology, Cincinnati Children's Hospital Medical Center, and Department of Pediatrics, University of Cincinnati College of Medicine, Cincinnati; ${ }^{6}$ E.M. Morgan, MD, MSCE, Associate Professor, Division of Rheumatology, Cincinnati Children's Hospital Medical Center, and Department of Pediatrics, University of Cincinnati College of Medicine, Cincinnati, Ohio, USA.

Address correspondence to Dr. E.A. Smitherman, The Children's Hospital, CPP N G10, 1600 7th Ave. S., Birmingham, AL 35223-1711, USA. Email:emily.smitherman@peds.uab.edu.

Accepted for publication August 15, 2019. significantly higher rates of active disease and irreversible organ damage $^{1}$ and a 2 -fold higher mortality rate ${ }^{2}$. Because treatment of cSLE often entails chronic use of glucocorticoids, it is critical to minimize the associated cardiovascular and bone health toxicities, including vitamin D deficiency ${ }^{3}$, hyperlipidemia ${ }^{4}$, and decreased bone mass ${ }^{5}$. Further, intervention to prevent adverse sequelae is critically important during childhood because of vulnerability with growth, development, and bone mass accrual and the potential for cumulative morbidity over time. There is evidence that improved quality of care in the rheumatology clinic is associated with improved clinical outcomes for adults with SLE ${ }^{6,7}$. To improve longterm morbidity and mortality in cSLE, dedicated work to define quality care and implement interventions to achieve care delivery standards is needed, followed by documented improvement of health outcomes.

To date, quality measures that emphasize preventive processes of care, including comorbidity screenings, have been developed for $\mathrm{CSLE}^{8}$. However, initial benchmarking at 7 international pediatric rheumatology centers revealed suboptimal performance and marked variation across the 26 quality indicators in clinical practice, especially for cardiovascular $(\mathrm{CV})$ and bone health

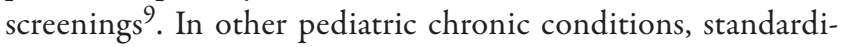

Personal non-commercial use only. The Journal of Rheumatology Copyright $\odot$ 2020. All rights reserved 
zation and reliable implementation of processes of care have led to significant improvements in clinical outcomes ${ }^{10,11}$. In a cohort of pediatric kidney transplant recipients, introducing previsit planning to reliably implement cholesterol monitoring led to a significant increase in the number of patients receiving statin therapy and with cholesterol values at goal ${ }^{12}$. Therefore, by designing a reliable system to improve rates of preventive screenings for patients with cSLE, earlier recognition and intervention on critical risk factors could have the potential to improve longterm health outcomes for this population.

The objective of our study was to design and implement an intervention to improve CV and bone health screenings in patients with cSLE. Published quality indicators were adapted to track performance at our local center for completing vitamin D and lipid profiles annually and bone mineral density testing with dual-energy $\mathrm{x}$-ray absorptiometry (DXA) at least once. The specific aim of the intervention was to increase the percentage of cSLE clinic visits with completed CV and bone health screenings from baseline to $>80 \%$ over the period from August 2016 to December 2017. We hypothesized that use of quality improvement methods enables the design and implementation of a healthcare system intervention to reliably improve the rate of comorbidity screenings, thereby improving the quality of care provided to our patients with cSLE.

\section{MATERIALS AND METHODS}

Context. This study took place in a pediatric rheumatology clinic at a large, Midwestern US pediatric tertiary care center that actively follows around 120 patients with cSLE, often into adulthood. Pediatric rheumatology clinical providers included 10 attending physicians, 2 with combined internal medicine-pediatrics training, and 1 nurse practitioner, with a mean of 15 years in practice (range $0.5-35$ ). The electronic health record (EHR) was used to identify patients through a disease registry function and to generate reports for screening completions and screening failures. Test results were later extracted from the EHR by both automated and manual review. The Institutional Review Board approved the study and waived the requirement for consent (study ID 2018-1455).

Intervention. The intervention was structured following the Model for Improvement ${ }^{13}$. We formed a team from pediatric rheumatology, including the lead investigator (EAS), a registered nurse, nurse coordinator, nursing director, quality improvement divisional leaders, and the division director. The team hypothesized key system drivers necessary to achieve reliable health screening for patients with cSLE, in keeping with quality guidelines (Figure 1). The current clinic process for cSLE disease management using previsit planning was thoroughly reviewed, theoretical and known failures at each step of the process were identified, and a list of potential interventions was developed (Figure 2).

The intervention began in August 2016 with presentation of 6 months of baseline performance data to clinical providers, and feedback was solicited on potential process interventions. Through a series of iterative test cycles to refine process improvements, we focused our intervention on an existing previsit planning procedure. Prior to the intervention, the rheumatology nurse coordinator or registered nurse would use the EHR to identify patients with cSLE who were scheduled for the clinic 1 week in advance and would review certain criteria that varied by provider. With the intervention, this previsit planning process was standardized so that for every patient, the EHR was reviewed for results of a serum $25(\mathrm{OH}) \mathrm{D}$ level and serum lipid profile during the preceding 410 days (allowing for a 45 -day margin past 1 year) and
1 prior DXA scan. If results were not found, electronic orders were entered and saved in the EHR for review and/or signoff by the treating provider during the upcoming clinic visit. The saved orders were documented in a note and were visible in the EHR order entry function; however, a chart could still be closed without the provider taking action if the provider did not navigate to the order entry screen or sign the saved orders during the visit.

During the initial 6 months of the intervention, the lead investigator received a weekly patient-level automated report for visits completed the previous week with the most recent completion date for each of the 3 screenings. If screenings were due but had not been completed, chart review was performed and a reason was recorded. Through this analysis, additional interventions were implemented to increase the reliability and scope of the previsit planning procedure, including standardizing previsit planning documentation, updating an existing electronic order set, expanding capability for electronic entry of results on tests performed external to the institution to make them searchable for inclusion in compliance reports, and training clinic staff on these processes.

Measures. The study population was defined as patients scheduled in the pediatric rheumatology clinic with the International Classification of Diseases-10-Clinical Modification diagnosis code of M32* for SLE used in at least 2 prior clinic visits in the pediatric rheumatology department and at least 1 month since diagnosis with no age exclusions. Patients were identified through a validated registry list in the EHR. The primary outcome measure was the percentage of cSLE clinic visits each month with CV and bone health screenings completed, including serum $25(\mathrm{OH}) \mathrm{D}$ in the last 410 days, serum lipid profile in the last 410 days, and 1 prior DXA scan. We reviewed 6 months of baseline data starting in February 2016, and then began prospective collection in August 2016. We retrospectively measured the proportion of the population with screenings completed during the baseline period and intervention period. We also collected the results of the screening tests and whether an action was taken by the care provider in response to abnormal results, such as a new medication prescription, new referral, followup testing, or documentation of counseling the patient and/or family. For screenings that were not completed, we recorded a reason related to the clinic process.

In a posthoc analysis, we explored the association of patient-level measures with successful screening completion (yes/no). These patient-level measures were (1) demographics including age, sex, and race; (2) clinical characteristics at the start of the intervention including disease duration in years, body mass index (BMI), SLE Disease Activity Index (SLEDAI) ${ }^{14}$, and Systemic Lupus International Collaborating Clinics/American College of Rheumatology Damage Index (SDI) ${ }^{15}$; and (3) health system factors including insurance status, number of clinic visits during the intervention period, whether all screenings were completed at baseline, and whether the patient's provider was a higher versus lower volume provider. To determine provider volume, the number of patients per provider during the intervention period was calculated, and providers were divided into patient volume quartiles. Lower volume providers were in the first and second quartiles, and higher volume providers were in the third and fourth quartiles.

Analysis. The intervention was designed as an interrupted time series trial, in which data are longitudinally collected before and after intervention ${ }^{13}$. The primary outcome measure was plotted over time on an attribute statistical process control chart, or P-chart, that includes a mean centerline and upper and lower control limits calculated using the $\mathrm{SD}^{16}$. This allows for distinguishing common cause variation within the system and special cause variation reflecting a change to the system. Based on established rules, we subgrouped the proportion of clinic visits in which screenings were completed before or by 1 week after the visit by month and adjusted the centerline and control limits to document special cause variation and improvement ${ }^{16}$. The goal was for $80 \%$ of visits each month to have completed screenings to achieve first-level process reliability ${ }^{17}$, and we tracked each

Personal non-commercial use only. The Journal of Rheumatology Copyright $\subset$ 2020. All rights reserved. 


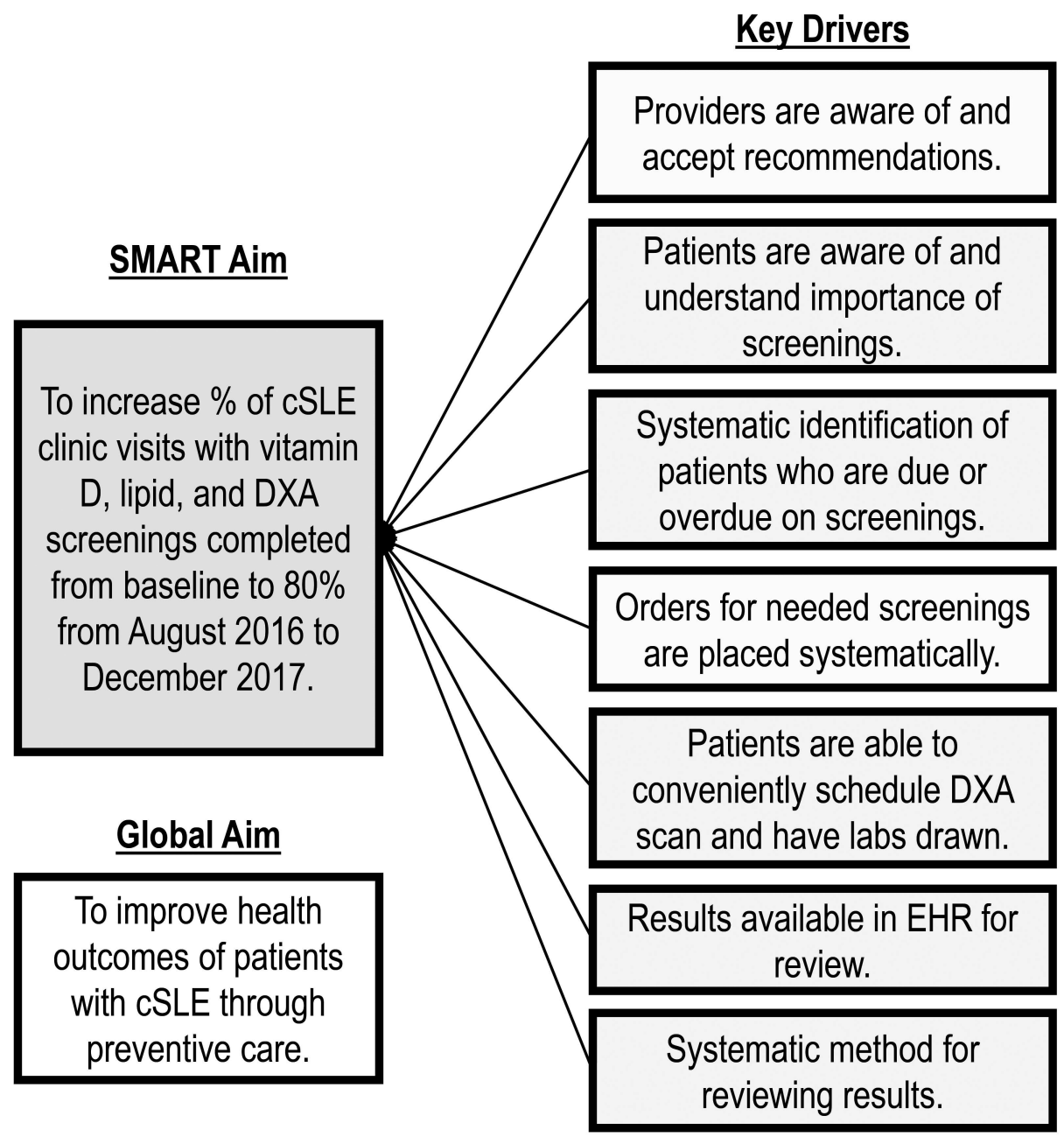

Figure 1. Key driver diagram for improving cardiovascular and bone health screenings for childhood-onset systemic lupus erythematosus (cSLE). SMART: specific, measurable, actionable, relevant, time-bound; DXA: dual-energy $\mathrm{x}$-ray absorptiometry; EHR: electronic health record.

screening separately and together as a bundle. These charts were generated using a standard package from Microsoft Excel.

In addition, for patients with visits during both the baseline and intervention period, we compared the completion rates for each of the 3 screening tests using McNemar's test for paired observations. We calculated the percentage of patients with abnormal screening results during the intervention and the percentage of abnormal results where an action was taken by the provider. We also calculated the average monthly results over time.

In the posthoc analysis, we divided patients into 2 groups based on whether all 3 screenings were completed by the end of the study and performed multiple univariate comparisons to identify differences in patient demographics, clinical characteristics, and health system factors between these 2 groups. Descriptive statistics were performed for each variable listed above using frequency and percentage for categorical variables and mean and SD for continuous variables, respectively. Group differences in categorical variables were analyzed using chi-square tests or Fisher's exact tests for small cell values, and continuous variables were analyzed using t tests. BMI was analyzed using Wilcoxon rank-sum test. Statistical significance was set at $\mathrm{p} \leq$ 0.05 (2-tailed). Analyses were performed using R software ${ }^{18}$.

\section{RESULTS}

During the 23-month study period, 123 individual patients were evaluated across 619 clinic encounters staffed by 11 pediatric rheumatology providers. Of these, 100 patients were seen during the baseline period from February 2016 through July 2016, and 111 were seen during the intervention period from August 2016 through December 2017. Eighty-eight were seen during both. The mean age for all patients was 19.5 years, with $86 \%$ female and $52 \%$ white (Table 1 ). The number of visits per patient during the total study period ranged from 1 to 13 with a median of 5 visits per patient. The number of patients followed by each provider ranged from 1 to 42 with a median of 5 patients per provider. Five providers were considered higher volume and 6 were considered lower volume based on the quartile definition described above.

At baseline, the proportion of patients with completed screenings was $54 \%$ for vitamin D, $55 \%$ for lipid, $57 \%$ for DXA, and only $22 \%$ for the bundle of all 3 tests. On each of the statis- 


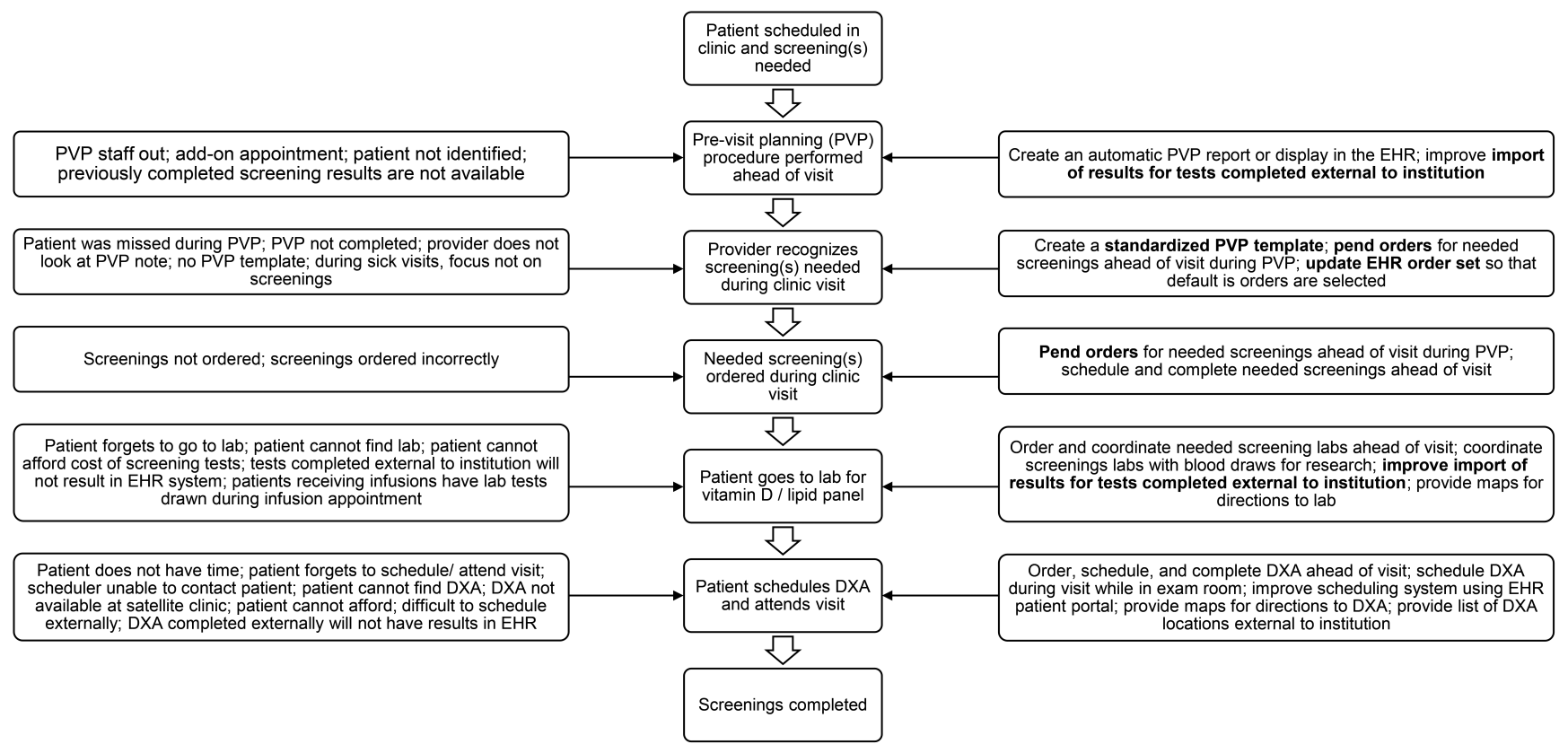

Figure 2. Simplified Failure Modes Effect Analysis for improving the reliability of performing cardiovascular and bone health screenings for cSLE. Interventions that were implemented are highlighted in bold. PVP: previsit planning; EHR: electronic health record; DXA: dual-energy x-ray absorptiometry; cSLE: childhood-onset systemic lupus erythematosus.

Table 1. Patient demographic characteristics.

\begin{tabular}{lc}
\hline Total $\mathrm{n}$ & 123 \\
\hline Age, yrs, mean (SD) & $19.5(4)$ \\
Female, $\mathrm{n}(\%)$ & $106(86)$ \\
Race, $\mathrm{n}(\%)$ & \\
$\quad$ White & $64(52)$ \\
African American & $48(39)$ \\
Asian & $7(6)$ \\
Hispanic & $2(2)$ \\
Other & $2(2)$ \\
\hline
\end{tabular}

tical process control P-charts that tracked performance of each screening separately and as a bundle, a shift was observed in the proportion of clinic visits each month with screenings completed, which corresponded to the start of the previsit planning intervention (Figure 3). This improvement was sustained for over a year. By the end of the intervention, the proportion of patients with completed screenings was $92 \%$ for vitamin D, $84 \%$ for lipid, $78 \%$ for DXA, and $64 \%$ for the bundle. For the 88 patients $(72 \%$ of total) seen during both the baseline and intervention period, there was a significant difference in the proportion with completed screenings for vitamin D (chi-square $=29.3$, $\mathrm{p}<0.01$ ), lipids (chi-square $=15.6, \mathrm{p}<0.01$ ), and DXA (chi-square $=17.1, \mathrm{p}<0.01)$. Improvement was observed across all providers.

During the intervention, 101 patients had vitamin D screenings completed, 88 had lipids completed, and 51 had a DXA scan (Table 2). A majority of patients had abnormal serum 25(OH)D levels, with $42(42 \%)$ classified as insufficient (20-30 $\mathrm{ng} / \mathrm{ml})$ and $23(23 \%)$ as deficient $(<20 \mathrm{ng} / \mathrm{ml})$. In addition, a majority of patients had an abnormal total body Z score on DXA with 21 (41\%) with Z score < - 1.0 SD and 8 (16\%) with Z score $<-2.0$. Although treatment algorithms were not part of the intervention, providers did respond to abnormal laboratory values in the majority of cases, including prescription of vitamin D supplementation and/or repeat testing for abnormal vitamin $\mathrm{D}$, lifestyle modification counseling and referrals to physical fitness and/or nutrition programs for abnormal lipids, and prescription of vitamin $\mathrm{D}$ and/or calcium and lifestyle modification counseling for abnormal DXA Z scores. There was improvement over time in the mean \pm SD $25(\mathrm{OH}) \mathrm{D}$ values collected each month from $27+12 \mathrm{ng} / \mathrm{ml}$ at baseline to $34.5 \pm 16 \mathrm{ng} / \mathrm{ml}$ at the end of the study period.

Reasons for screenings not completed during the intervention period were identified by review of the EHR and tracked using Pareto charts ${ }^{16}$. The majority of vitamin D non-completions ( 5 of 9) occurred when previsit planning was not done, such as with urgent visits (i.e., scheduled outside of the regular previsit planning window). Six of 17 lipid non-completions were also due to urgent scheduling, and 5 of 17 to a glitch discovered in releasing electronic orders in phlebotomy. For DXA scans, 7 of 23 non-completions occurred when providers did not sign the saved orders for various documented reasons such as lack of insurance, and an additional 7 of 23 were not done because of

\section{Personal non-commercial use only. The Journal of Rheumatology Copyright @ 2020. All rights reserved.}



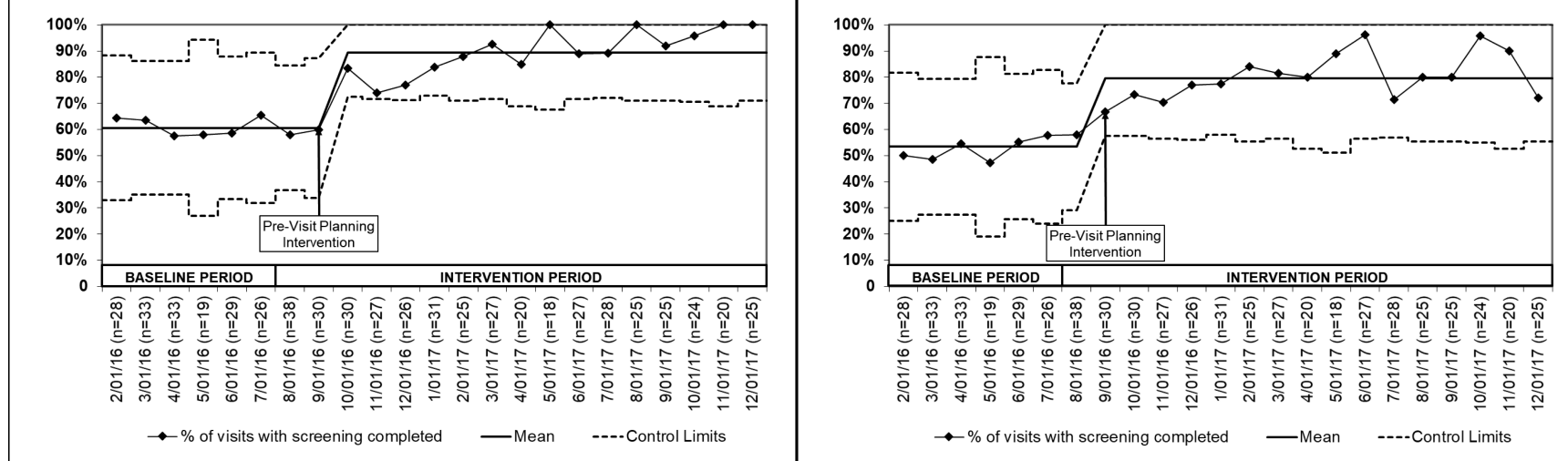

C $\quad$ of Visits with DXA Screening Completed
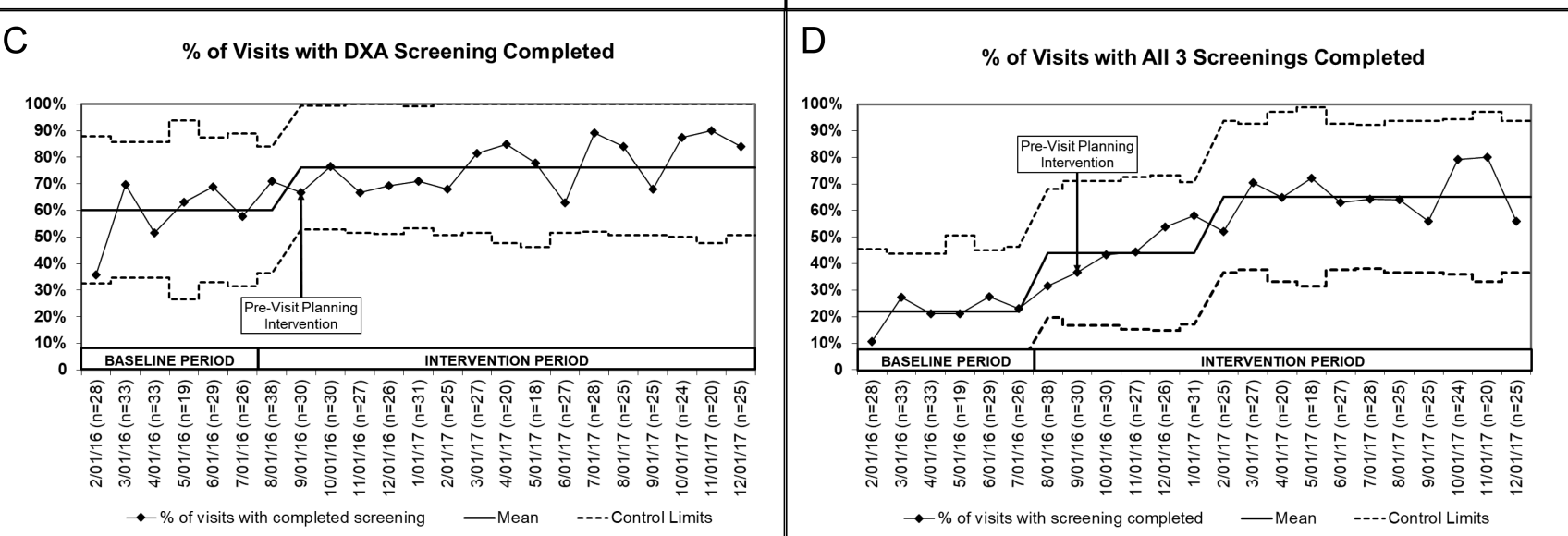

Figure 3. Statistical process control P charts showing the percentage of cSLE clinic visits with screenings completed for $(A)$ annual serum $25(\mathrm{OH}) \mathrm{D}$, (B) annual serum lipid profile, (C) 1-time DXA scan, and (D) the bundle of all 3 screenings. cSLE: childhood-onset systemic lupus erythematosus; DXA: dual-energy x-ray absorptiometry.

Table 2. Results of serum $25(\mathrm{OH}) \mathrm{D}$, serum lipid profiles, and DXA scan screenings completed during the intervention.

\begin{tabular}{lcccc}
\hline Variables & $25(\mathrm{OH}) \mathrm{D}$ & Total Cholesterol & LDL & DXA \\
\hline Patients with screening completed, $\mathrm{n}$ & 101 & 88 & 88 & 51 \\
Average result, mean (SD) & $28(12)$ & $151(40)$ & $73(33)$ & $-0.7(1.2)$ \\
Abnormal results, $\mathrm{n}(\%)^{*}$ & $65(64)$ & $10(11)$ & $7(8)$ & $29(57)$ \\
Action on abnormal result, $\mathrm{n}(\%)$ & $43(66)$ & $6(60)$ & $5(71)$ & $22(76)$ \\
\hline
\end{tabular}

${ }^{*}$ Definitions for abnormal results: $25(\mathrm{OH}) \mathrm{D}<30 \mathrm{ng} / \mathrm{ml}$, total cholesterol $>200 \mathrm{mg} / \mathrm{dl}, \mathrm{LDL}>130 \mathrm{mg} / \mathrm{dl}$, DXA total body $\mathrm{Z}$ score <-1.0 SD. DXA: dual-energy $\mathrm{x}$-ray absorptiometry; LDL: low-density lipoprotein.

scheduling difficulties. The other reasons for non-completion across all 3 screenings included tests ordered external to our hospital system without documentation of results in the EHR, patients failing to present to the phlebotomy unit after the clinic visit despite instructions, and patients who received laboratory draws during scheduled infusion visits because of a parallel but separate process used for infusion orders outside of the usual clinic workflow, through which saved orders cannot be visualized.

Finally, multiple patient-level variables were compared in a posthoc analysis between patients in the intervention group who had all 3 screenings completed $(\mathrm{n}=71,64 \%)$ versus not all completed $(n=40,36 \%)$ at the end of the study period. Patients with all 3 screenings completed had higher SLEDAI scores, higher SDI scores, and more clinic encounters, and were more likely to have had screenings completed prior to the intervention (Table 3). Differences were not observed in age, sex, race, disease duration, BMI, insurance status, or the provider's patient volume status.

\section{DISCUSSION}

This study is the first to our knowledge to demonstrate improvement in completing $\mathrm{CV}$ and bone health screenings for 
patients with cSLE. We used rigorous quality improvement methodology to design and implement a health system intervention that improved performance for quality of care measures that were previously benchmarked as suboptimal ${ }^{9}$. In addition to sustained improvement in screening completion, our study also demonstrated provider response to the majority of abnormal results without use of a treatment algorithm and improvement in vitamin $\mathrm{D}$ results over time. By developing a reliable system of care that emphasizes early risk-based monitoring and intervention, there is potential to significantly improve longterm comorbidity outcomes for patients with cSLE.

The results of our baseline assessment of completed screenings were within the range of previously published benchmarks of $\mathrm{CV}$ and bone health quality indicators from an international sample of pediatric rheumatology centers ${ }^{9}$, in addition to other single-center studies ${ }^{18,19}$. Through the application of quality improvement methods, we were able to demonstrate sustained improvement in these measures that resulted in a change to our routine provision of care for patients with cSLE within our local clinic. The use of an interrupted time series design and statistical process control charts for measurement allowed us to determine that our intervention led to process improvement. Notably, this improvement was sustained for over a year, accounting for any potential seasonal effects.

The key feature in our intervention that led to improvement was the use of a standardized system to review patient data ahead of scheduled clinic visits and to prepare for identified needs during the clinic visit, a process referred to as previsit planning.
Rooted in the elements of the Chronic Care Model ${ }^{20}$, previsit planning is recognized as an important strategy to improve processes of care and outcomes for multiple chronic conditions, including in pediatrics ${ }^{10,12,21}$. Interviews of young adults with cSLE have previously revealed that comprehensive and coordinated care was a top healthcare priority ${ }^{22}$. However, active provision of non-visit care is resource-intense and may be challenging to implement in certain clinical contexts. The previsit planning intervention implemented in our study required the time and knowledge of highly skilled staff to conduct the previsit chart review and save orders for providers as well as sophisticated clinical information system capabilities of the existing EHR. While reliance on trained personnel is a limitation of the reported intervention, further automation is now being pursued because the system has proven stable ${ }^{13}$. We did not explicitly measure time spent on previsit review as a balancing measure because tasks were incorporated into an existing process, but this is an important consideration for replication in other settings. Such a highly technology-based solution affects the generalizability to care centers with limited EHR capability, but provides evidence for advocating for further investment in health information technology. Finally, while this process intervention limits the ability to comment on the distal effect on patient outcomes, by implementing a reliable risk assessment process and demonstrating documentation of provider response, we can infer an eventual downstream effect.

An interesting finding in our posthoc analysis was that patients who had all screenings completed at the end of the study had

Table 3. Comparison of patients in the intervention, $\mathrm{n}=111$, with all 3 screenings completed versus not all completed at end of study.

\begin{tabular}{lccc}
\hline Characteristics & $\begin{array}{c}\text { Group with All 3 Screenings } \\
\text { Completed, } \mathrm{n}=71\end{array}$ & $\begin{array}{c}\text { Group with Not All } \\
\text { Screenings Completed, } \mathrm{n}=40\end{array}$ & $\mathrm{p}$ \\
\hline Demographic characteristics & & & \\
Age, yrs, mean (SD, range) & $19.7(4.1,13-40)$ & $19.0(3.8,12-28)$ & 0.351 \\
Female, $\mathrm{n}(\%)$ & $62(87)$ & $33(83)$ & 0.679 \\
White, $\mathrm{n}(\%)$ & $37(52)$ & $18(45)$ & 0.602 \\
Clinical characteristics & $4.7(3.8)$ & $4(3.3)$ & 0.317 \\
Disease duration, yrs, mean (SD) & $24.3(4.9)$ & $26.2(7.8)$ & 0.502 \\
BMI, kg/m ${ }^{2}$, mean (SD) & $4.04(5.7)$ & $2.08(2.4)$ & $0.013^{*}$ \\
SLEDAI, mean (SD) & $0.73(1.2)$ & $0.38(0.56)$ & $0.042^{*}$ \\
SDI, mean (SD) & & & \\
Health system characteristics & $39(55)$ & $19(48)$ & 0.687 \\
Insurance status, $\mathrm{n}(\%)$ & $26(37)$ & $18(45)$ & \\
Private & $6(8)$ & $3(8)$ & $0.016^{*}$ \\
Public & $4.5(2.7)$ & $3.3(2.4)$ & $0.024^{*}$ \\
None & $18 / 59(31)$ & $34(85)$ & 0.716 \\
Clinic visits per patient, mean (SD) & $57(80)$ & & \\
All screenings complete at baseline, $\mathrm{n}(\%)$ & & & \\
Higher volume provider, $\mathrm{n}(\%)$ & & & \\
\hline
\end{tabular}

\footnotetext{
* Denotes significance. Comparisons for categorical variables were performed using chi-square test except insurance status and all screenings complete at baseline used Fisher's exact test; comparisons for continuous variables were performed using Student $\mathrm{t}$ test; comparisons for BMI were performed using Wilcoxon rank-sum test. Patients not followed during the baseline period were not included in the analysis of all screenings complete at baseline. BMI: body mass index; SLEDAI: Systemic Lupus Erythematosus Disease Activity Index; SDI: Systemic Lupus International Collaborating Clinics/American College of Rheumatology Damage Index.
} 
more clinic visits during the intervention period compared to those who did not have all screenings completed. Previous studies in SLE have also reported an association between number of clinical visits and improved performance on quality indicator measures 5 . It may be that more clinic visits leads to more opportunities to place the order for needed screenings or to discuss and mitigate potential barriers to completing screenings. In considering fewer clinic visits in the group of patients with not all screenings completed, this may reflect the time constraints encountered when visits occur infrequently in cSLE. Infrequent appointments require catchup on a large number of complex chronic illness care maintenance items in a constrained period of time (e.g., $30 \mathrm{~min}$ ), and when such visits are made urgently rather than preventively, the acute illness may supersede health maintenance concerns. This finding advocates for more emphasis on non-visit methods of care provision for patients with chronic conditions, such as a care manager or care coordinator. Although the patients with completed screenings also had higher disease activity scores and damage indices at the start of the intervention, which may account for the increased number of visits, this observation may suggest an increased vigilance for health maintenance in patients with more severe disease. Finally, this finding may indicate that patients who more readily engage with the healthcare system, whether because of severe disease or otherwise, are more likely to receive high-quality care. If this hypothesis is true and especially if this affects longterm outcomes, design of a behavioral intervention to target patient engagement in their healthcare would be of high value.

While the aim of our study was to design and implement a single-center intervention, there is considerable potential to spread the improvement to other centers. Use of previsit planning to identify and mitigate patient needs prior to the clinic visit is a well-accepted tool to improve care for patients with chronic conditions and would be amenable for testing across multiple centers to enhance the learning and potential effect of the intervention. A multicenter implementation network, Pediatric Rheumatology Care and Outcomes Improvement Network (PRCOIN), was established in 2011 to improve the care and outcomes for children with rheumatic conditions ${ }^{23}$. A substantial effort through PR-COIN to date has focused on designing and implementing previsit planning strategies for patients with juvenile idiopathic arthritis (JIA). Because all pediatric rheumatology centers participating in PR-COIN care for patients with JIA and cSLE, our study demonstrates the utility of expansion of previsit planning to patients with cSLE. The established infrastructure of PR-COIN could spur the testing and spread of our cSLE comorbidity screening intervention at a multicenter level, to improve more patient health outcomes.

We developed an intervention using care coordination and health information technology that significantly improved the proportion of cSLE patients with completed CV and bone health comorbidity screenings. The design of our intervention could be applied to other categories of health maintenance monitoring both for cSLE and other chronic conditions. In addition, further investi- gation into the effect of patient engagement with the healthcare system on quality of care and health outcomes should be considered. This intervention serves as an initial step toward a comprehensive management approach to improve quality of care in cSLE. We assert that reliable completion of preventive screenings for patients with cSLE will ultimately lead to lower rates of comorbidities in adulthood and improve longterm outcomes for this high-risk population.

\section{ACKNOWLEDGMENT}

The authors acknowledge the efforts of Courtney Paffett, RN, BSN, CPN, and Barbara Weyer, RN, MSN, in making important contributions to the improvement work. We also acknowledge the participation of all pediatric rheumatology clinical providers and staff in the William S. Rowe Division of Rheumatology at Cincinnati Children's Hospital Medical Center. Finally, we acknowledge the analytic support provided by Amy M. Anneken, MS, as well as the contribution of multiple faculty and staff through the James M. Anderson Center for Health Systems Excellence's Intermediate Improvement Science Series course.

\section{REFERENCES}

1. Brunner HI, Gladman DD, Ibanez D, Urowitz MD, Silverman ED Difference in disease features between childhood-onset and adult-onset systemic lupus erythematosus. Arthritis Rheum 2008; 58:556-62.

2. Hersh AO, Trupin L, Yazdany J, Panopalis P, Julian L, Katz P, et al. Childhood-onset disease as a predictor of mortality in an adult cohort of patients with systemic lupus erythematosus. Arthritis Care Res 2010;62:1152-9.

3. Robinson AB, Tangpricha V, Yow E, Gurion R, McComsey GA, Schanberg LE, et al. Vitamin D deficiency is common and associated with increased C-reactive protein in children and young adults with lupus: an atherosclerosis prevention in pediatric lupus erythematosus substudy. Lupus Sci Med 2014;1:e000011.

4. Ardoin SP, Sandborg C, Schanberg LE. Management of dyslipidemia in children and adolescents with systemic lupus erythematosus. Lupus 2007;16:618-26.

5. Compeyrot-Lacassagne S, Tyrrell PN, Atenafu E, Doria AS, Stephens D, Gilday D, et al. Prevalence and etiology of low bone mineral density in juvenile systemic lupus erythematosus. Arthritis Rheum 2007;56:1966-73.

6. Yazdany J, Trupin L, Schmajuk G, Katz PP, Yelin EH. Quality of care in systemic lupus erythematosus: the association between process and outcome measures in the lupus outcomes study. BMJ Qual Saf 2014;23:659-66.

7. Yelin E, Yazdany J, Trupin L. Relationship between process of care and a subsequent increase in damage in systemic lupus erythematosus. Arthritis Care Res 2017;69:927-32.

8. Hollander MC, Sage JM, Greenler AJ, Pendl J, Avcin T, Espada G, et al. International consensus for provisions of quality-driven care in childhood-onset systemic lupus erythematosus. Arthritis Care Res 2013;65:1416-23.

9. Mina R, Harris JG, Klein-Gitelman MS, Appenzeller S, Centeville $\mathrm{M}$, Eskra D, et al. Initial benchmarking of the quality of medical care in childhood-onset systemic lupus erythematosus. Arthritis Care Res 2016;68:179-86.

10. Crandall WV, Margolis PA, Kappelman MD, King EC, Pratt JM, Boyle BM, et al. Improved outcomes in a quality improvement collaborative for pediatric inflammatory bowel disease. Pediatrics 2012;129:e1030-41. 
11. Anderson JB, Beekman RH 3rd, Kugler JD, Rosenthal GL, Jenkins $\mathrm{KJ}$, Klitzner TS, et al. Use of a learning network to improve variation in interstage weight gain after the Norwood operation. Congenit Heart Dis 2014;9:512-20.

12. Hooper DK, Kirby CL, Margolis PA, Goebel J. Reliable individualized monitoring improves cholesterol control in kidney transplant recipients. Pediatrics 2013;131:e1271-9.

13. Langley G, Moen R, Nolan K, Nolan T, Norman C, Provost L. The improvement guide: a practical approach to enhancing organizational performance. 2nd ed. San Francisco: Jossey-Bass; 2009.

14. Bombardier C, Gladman DD, Urowitz MB, Caron D, Chang CH. Derivation of the SLEDAI. A disease activity index for lupus patients. The Committee on Prognosis Studies in SLE. Arthritis Rheum 1992;35:630-40.

15. Gladman D, Ginzler E, Goldsmith C, Fortin P, Liang M, Urowitz $\mathrm{M}$, et al. The development and initial validation of the Systemic Lupus International Collaborating Clinics/American College of Rheumatology Damage Index for systemic lupus erythematosus. Arthritis Rheum 1996;39:363-9.

16. Provost L, Murray $S$. The health care data guide: learning from data for improvement. 1st ed. San Francisco: Jossey-Bass; 2011.

17. Resar RK. Making noncatastrophic health care processes reliable: learning to walk before running in creating high-reliability organizations. Health Serv Res 2006;41:1677-89.
18. Harris JG, Maletta KI, Kuhn EM, Olson JC. Evaluation of quality indicators and disease damage in childhood-onset systemic lupus erythematosus patients. Clin Rheumatol 2017;36:351-9.

19. Basiaga ML, Burrows EK, Denburg MR, Meyers KE, Grossman AB, Mamula $P$, et al. Variation in preventive care in children receiving chronic glucocorticoid therapy. J Pediatr 2016; 179:226-32.

20. Wagner EH, Austin BT, Davis C, Hindmarsh M, Schaefer J, Bonomi A. Improving chronic illness care: Translating evidence into action. Health Affairs 2001;20:64-78.

21. Boyle MP, Sabadosa KA, Quinton HB, Marshall BC, Schechter MS Key findings of the US Cystic Fibrosis Foundation's clinical practice benchmarking project. BMJ Qual Saf 2014;23 Suppl 1:i15-22.

22. Tunnicliffe DJ, Singh-Grewal D, Craig JC, Howell M, Tugwell P, Mackie F, et al. Healthcare and research priorities of adolescents and young adults with systemic lupus erythematosus: a mixed-methods study. J Rheumatol 2017;44:444-51.

23. Harris JG, Bingham CA, Morgan EM. Improving care delivery and outcomes in pediatric rheumatic diseases. Curr Opin Rheumatol 2016;28:110-6. 